\title{
Dr Dick Zeilstra on the AxiaLIF
}

\section{—By Samo Fokter}

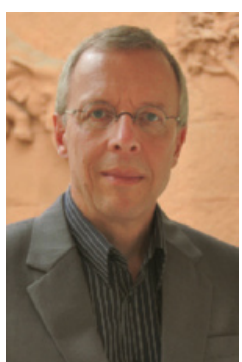

D. Zeilstra

ArgoSpine News and Journal: Minimal invasiveness is certainly a hot topic in spine surgery and AxiaLIF procedure is considered to be truly minimally invasive. When and how did you get familiar with the procedure?

Dick Zeilstra: I first saw the AxiaLIF technique in a presentation at a meeting in Snowbird in early February 2006. I immediately realized it was a technique that would make me independent of an access surgeon for anterior treatment of L5/S1. Till then I had been doing many disc replacements including L5/S1 and there was a long waiting list. Over time I had also begun to realize that there is not much added value in retaining motion at the L5/S1 level and that patients were just as well off with a fusion. My first cases were patients taken off the waiting list for disc replacement, after of course discussing the options with them.

ASN\&J: In your opinion, who is the best candidate for the procedure?

D.Z:: The indications are any case where you would like to fuse L5 with S1, so basically the same indications are for other techniques of fusion. We are not discussing a treatment, just a different technique and approach. Adding an AxiaLIF in spondylolisthesis cases provides for great resistance against shear forces.

ASN\&J: When I visited your department in Zwolle in 2008, I noticed that you keep all your patients on a file to carefully follow their functional recovery after surgery. How many were treated and what are the results?

D.Z.: Up to now 186 patients have been operated with the AxiaLIF technique. The majority (148) are patients with a single level symptomatic discopathy causing back pain.

ASN\&J: How do you evaluate fusion, at what follow-up time do you usually confirm it?

D.Z.: Standard follow up includes a CT scan after one year. Up till now 123 patients have had a CT scan with a definite fusion in $91 \%$ of the cases. There were 2 clear cases of non-union, in the remaining patients the fusion was unclear, but most of these were clinically doing well.

ASN\&J: Could you please explain us your tips and pearls about the primary procedure in details?

D.Z.: An important point is pre-operative planning and checking for feasibility. Careful preparation along the sacrum and exact docking of the K-wire will guarantee a good positioning of the rod. Of course meticulous disc tissue removal and scraping of the endplates is important for the fusion. Distraction is almost never indicated, it may even lead to subsidence and violates Wolf's law. Adding of facet screws increases stability and leads to better fusion rates, whereas pedicle screws seem somewhat of an overkill.

ASN\&J: No spinal procedure is without risk and complication. What complications did you observe and how are you trying to avoid them in the future?

D.Z.: I have seen two acute abdomens. One was a suspected bowel perforation, and although this was treated as such by the surgeon on night duty it was not proven. In the second case a better evaluation was done and this proved to be a presacral haematoma. This was first managed conservatively, but due to persisting symptoms it was (easily) removed with immediate relief of symptoms.

ASN\&J: Did you ever have to remove the TranS1 screw and which approach would you recommend for this procedure?

D.Z.: I had one case of a spondylolisthesis where an AxiaLIF was added after reduction. As often seen in these cases, the sacral slope was very steep and therefore the rod not in the normal position but more oblique, pointing posteriorly. In L5 the rod was just behind the posterior wall of the vertebral body. The patient had a fall and the reduction slipped back, causing the rod to break through the posterior wall of L5. The patient was asymptomatic, the diagnosis was made on routine X-ray. After the level had adequately fused the rod was removed, which was easily done through the same approach. In cases of non union I just leave the rod where it is, there are hardly any good reasons to remove it There is still enough room for pedicle screws. As long as the rod can be reached it is however easy, but once bone is covering the sacral hole it may become problematic but then again in such cases hardly ever necessary.

ASN\&J: Have we looked at the topic from all aspects?

D.Z:: There is a lot to say about the technique. One thing I have learnt is that our biggest fear, the bowel, is hardly ever a real threat. Once the technique is mastered it is a very effective, safe and quick method to do a 360-fusion in less than an hour.

\section{(1) ... there is not much added value in retaining motion at the L5/S1 level...."}

